DOI: https://doi.org/10.35560/jcofarts 101/227-242

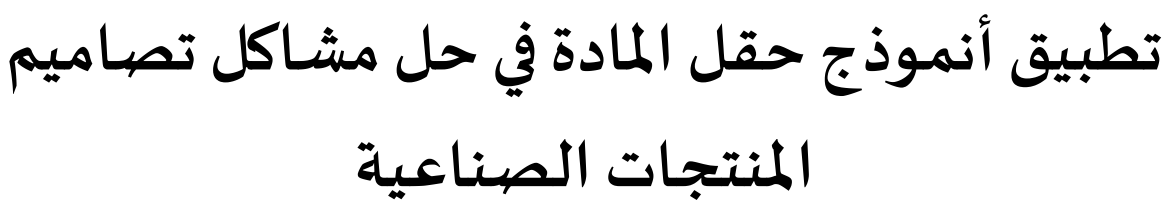

محمد علي حسين القيسي 1

ISSN(Online) 2523-2029, ISSN(Print) 1819-5229 مجلة الأكاديمي-العدد 101-السنة 2021

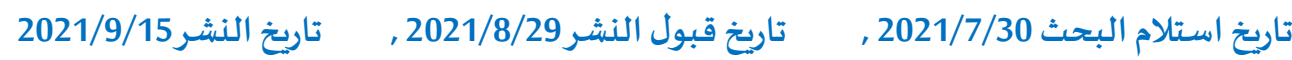

(c) (7) This work is licensed under a Creative Commons Attribution 4.0 International License

ملخص البحث

تساهم طرق وآليات حل المشكلات في تسهيل حياة الإنسان من خلال توفير أدوات لحل المشاكل اليومية

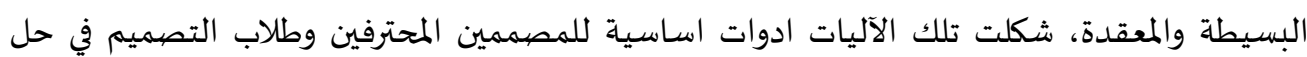
المشاكل التصيميمية.

تناول هذا البحث واحده من تلك الآليات وهو نموذج (حقل المادة) اذ يذكر ان هذه الآلية تتصف

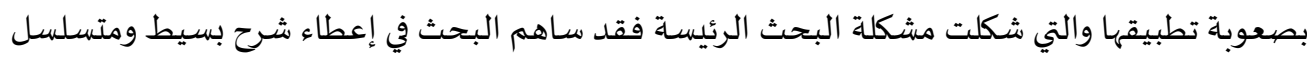
لتطبيق هذه الآلية اضافة الى تطبيقها لحل مشكلة قائمة و هي الصعوبات التي تواجه مستخدمى المجارف

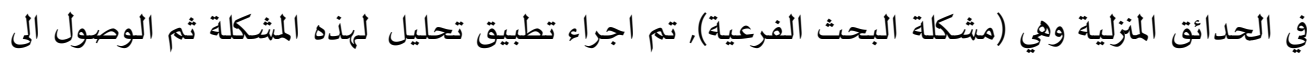
حل لمعالجتها وقد استخدم الباحث برنامج (3dsmax) لتنفيذ التصيميم المقترح. وكان اهم نتائج البحث: .

1- إن منهجية البحث وطريقة تطبيق آلية حقل المادة لحل مشكلة من الممكن أن تطبق لحل المشاكل التصميمية المختلفة البسيطة منها والمعقدة من قبل المصهمين المحترفين اضافة الى اهميته بالنسبة إلى طلاب التصيميم بشكل خاص. 2- قدم البحث نموذج مقترح لحل مشكلة المجرفة باستخدام آلية حقل المادة.

الكلمات الافتتاحية: حقل المادة، المجرفة، نظرية تريز

mohammed.ali@cofarts.uobaghdad.edu.iq . 1 كلية الفنون الجميلة/ جامعة بغداد 
تطبيق أنموذج حقل المادة في حل مشاكل تصياميم المنتجات الصناعية.........محمد علي حسين القيسي

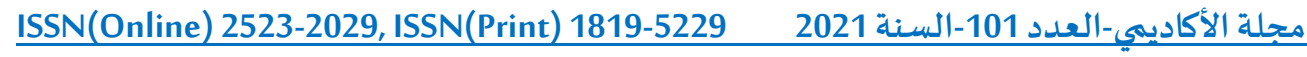

مقدمة

يُعِد حل المشكلات البسيطة والمعقدة التي تواجه الإنسان خلال مسيرته التطورية من الأمور المهمة في توفير الراحة والأمان.

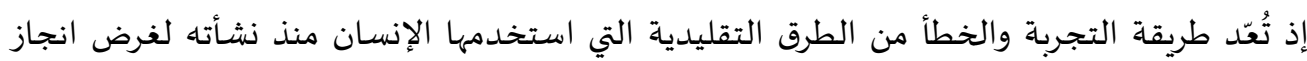

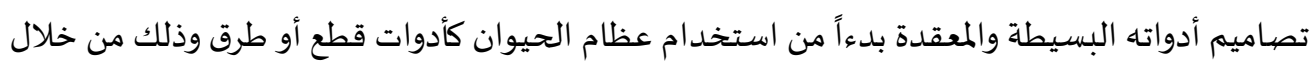
تجربة أنواع العظام المختلفة لاختيار ايهما الافضل في عمل الأدة للوظيفة المطلوبة. على الرغم من كون طريقة التجربة والخطأ في حل المشكلات هي من الطرق البسيطة والقديمة جداً والتي

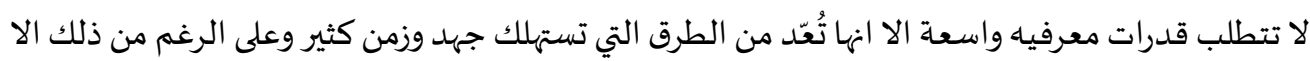

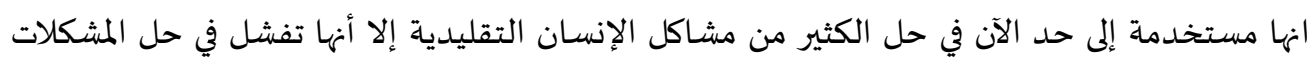

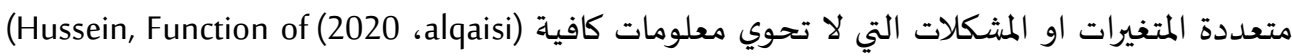
color in industrial products, 2010) إن العجز الذي أظهرت هذه الآلية اضافة الى تنوع وتعقيد الاحتياجات الإنسانية وتطور المعرفة المتزايد

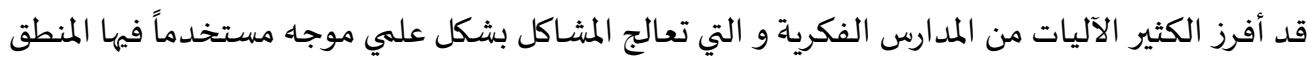

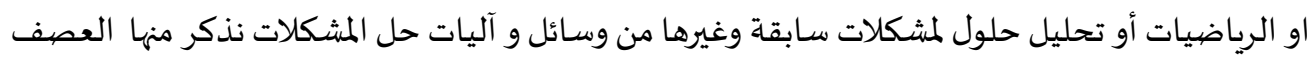
الذهني, (تريز), التسعة شبابيك, التحليل الوظيفي, المثالية والناتج النهائي المثالي وغيرها (Altshuller)،

(Alqaisi, 2011)

أما في ما يخص بحثنا هذا فسوف نتطرق إلى واحدة من الطرق الحديثة في حل المشكلات البسيطة

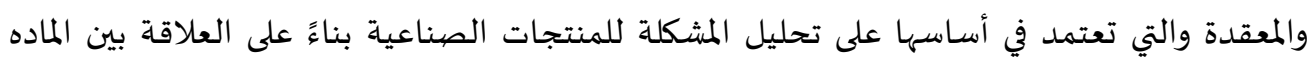

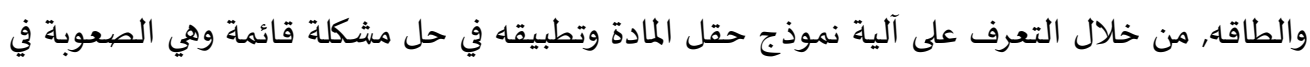

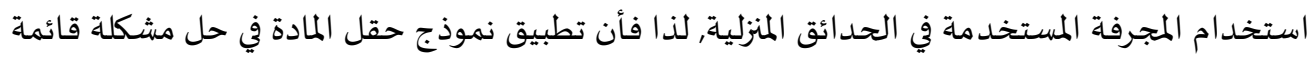

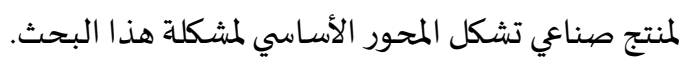

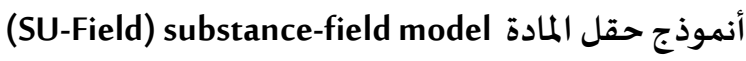

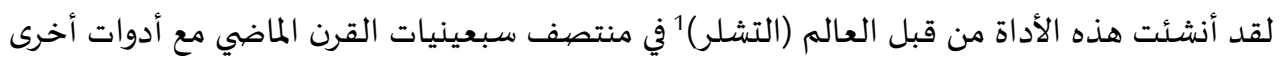

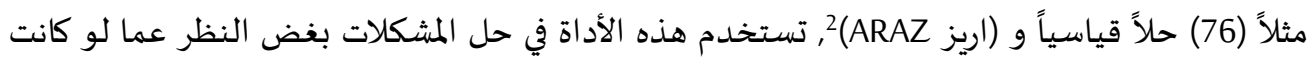

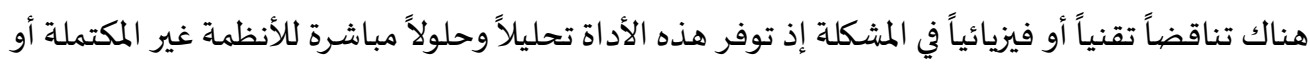

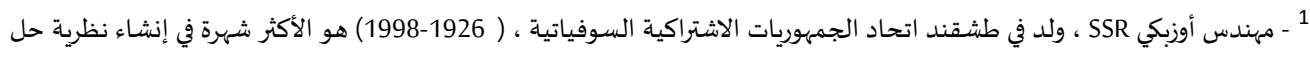

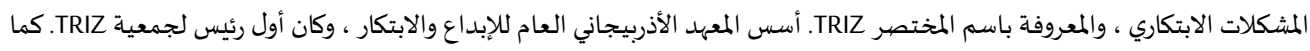

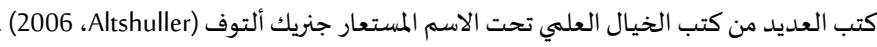

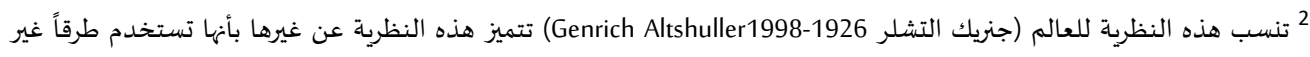

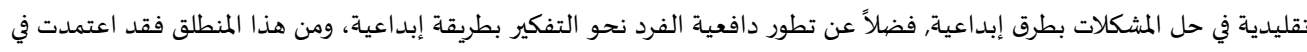

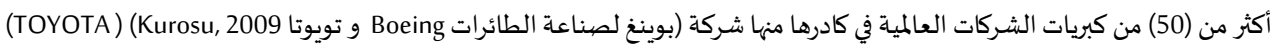


تطبيق أنموذج حقل المادة في حل مشاكل تصياميم المنتجات الصناعية.........محمد علي حسين القيسي ISSN(Online) 2523-2029, ISSN(Print) 1819-5229 2021 مجلة الأكاديي-العدي 101-السينة غير الكفوءة أو في حالة وجود أضرار في النظام أو في حل مشكلات التقييس والسيطرة النوعية و مشكلات

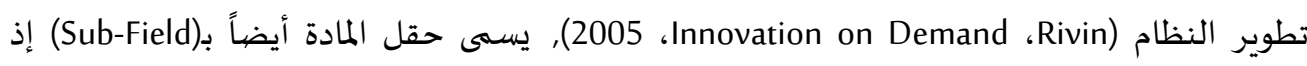
تستخدم بشكل دقيق لأعطاء تحليل مفصل للمشكلة ضمن النظام التقني القائم, هذا الوصف والتحليل

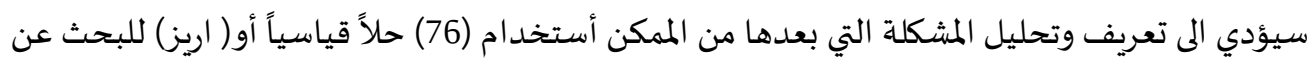

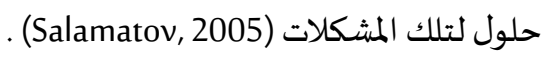
تستخدم هذه الأداة بشكل واسع من قبل مستخدمي وخبراء (تريز) التقليدية إلا إنها لا تكون من السهولة من قبل المستخدمين الأخرين إذ قلما تستخدم من قبل الأشخاص غير المتمرسين بشكل عالٍ بنظرية (تريز) وذلك لصعوبتها إذ تُعد هذه الأداة و(اريز) هما المسؤولان عن نظرة الآخرين إلى نظرية (تريز)

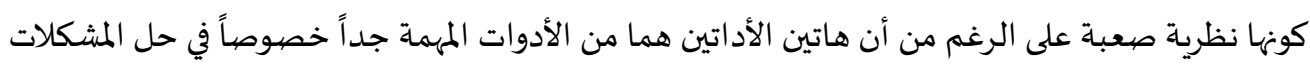

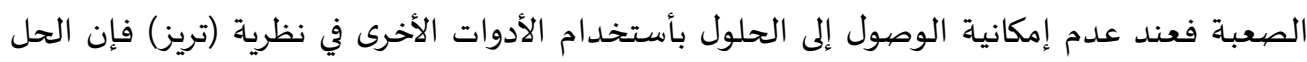

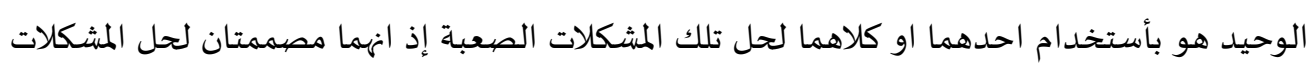

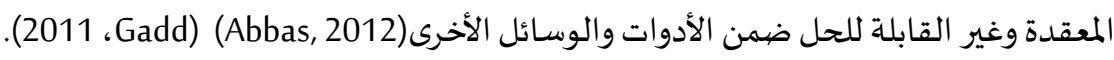
يتم تمثيل حقل المادة (Su-Field) على شكل مثلث أنظر الشكل (1) والذي هو عبارة عن رسم مبسط

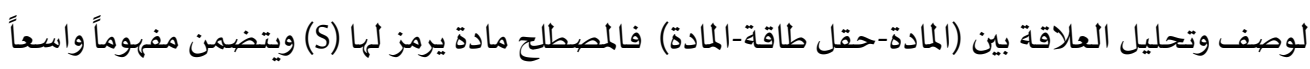

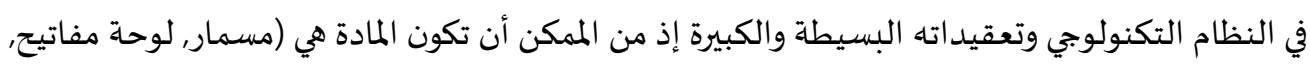

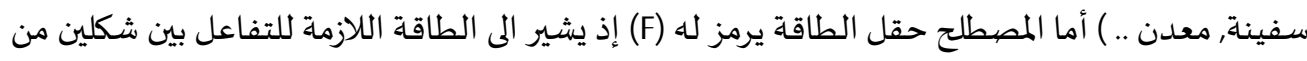

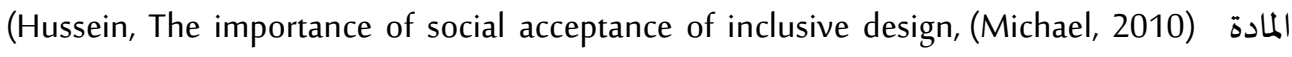

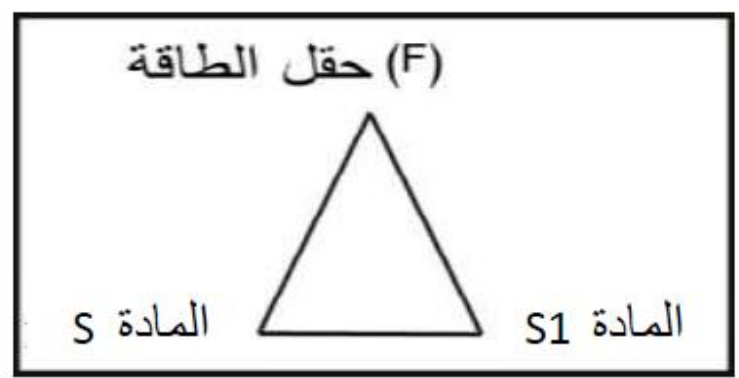

شكل(1) ترجمة الباحث

(2010، Michael)

أن مفهوم حقل الطاقة في نظرية (تريز) يختلف عنه في مفهوم الحقل في الفيزياء الحديثة فالأخيرة تحدد حقول الطاقة لأربعة حقول رئيسة وهي: 1. الجاذبية (والتي تكون موجودة بين شيئين أو أكثر).

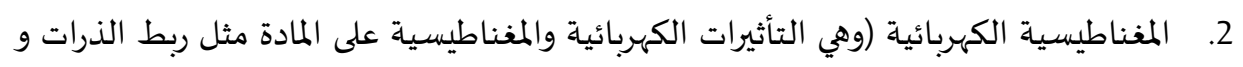

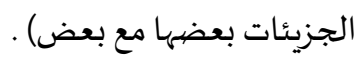


تطبيق أنموذج حقل المادة في حل مشاكل تصياميم المنتجات الصناعية.........محمد علي حسين القيسي ISSN(Online) 2523-2029, ISSN(Print) 1819-5229 2021 مجلة الأكاديي-العدد 101-السينة

$$
\text { 3. حقل الطاقة الذري الضعيف. }
$$

4. حقل الطاقة الذري القوي (هذا الحقل مسؤول عن الترابط بين نواة الذرات بعضهها مع بعض) . (others, 2006)

إِن هذا التقسيم لحقول الطاقة في الفيزياء ليس دائماً كافٍ عند حل مشكلات تصميمية في العلوم الهندسية ومفهوم الطاقة في نظرية (تريز) مختلف تماماً عن المفهوم في الفيزياء الحديثة إذ أن مفهوم (تريز) تمكن الشخص من التميز والتوصيف بحقل الطاقة بسهولة من خلال معرفة التفاعلات المختلفة بين المواد, وبما أن مجال عمل نظرية (تريز) هو في الحقل الهندسي والذي يتفرع الى حقول ( الميكانيك, الحراري,

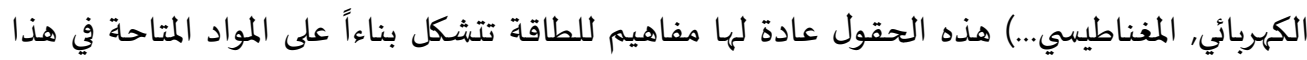

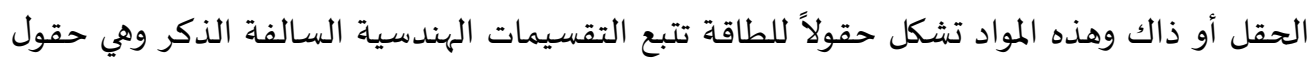

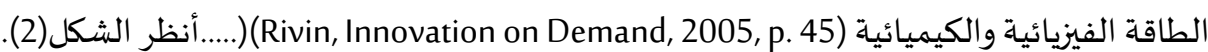

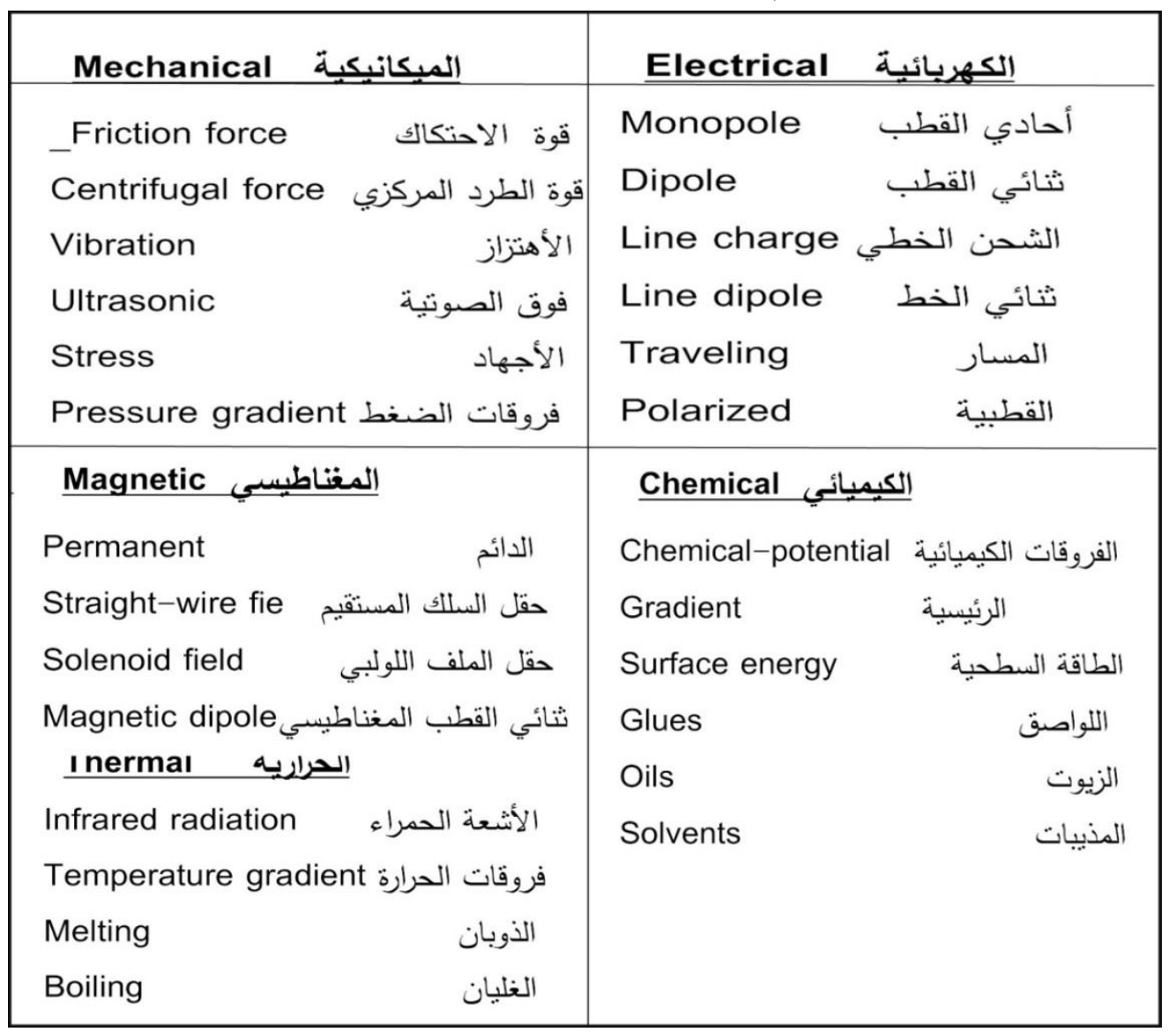

(2011, p. 49) الشكل(2) ترجمة الباحث

أما المادة (Substance) فيشار إليها في مثلث حقل المادة للرمز (S1) فيما تكون الأداة (S2) فيكون

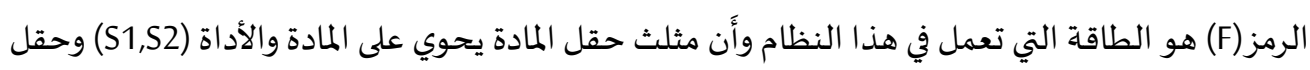


تطبيق أنموذج حقل المادة في حل مشاكل تصياميم المنتجات الصناعية.........محمد علي حسين القيسي ISSN(Online) 2523-2029, ISSN(Print) 1819-5229 2021 مجلة الأكاديي-العدد 101-السينة التفاعل بينهم وهو (F) فضلاً عن هذه الرموز هناك عدد من الرموز المختلفة المستخدمة في تمثيل مخطط

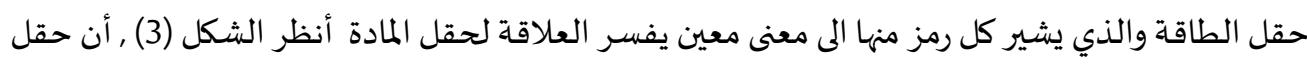

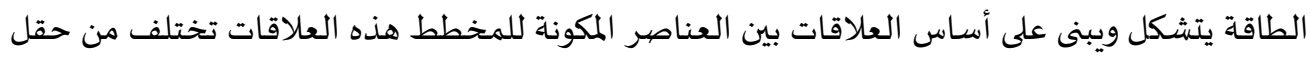

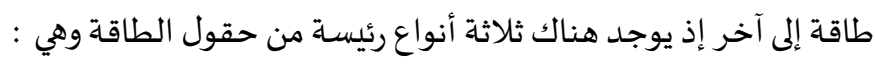
مادة تولد حقل طاقة (أنظر شكل A4). حقل طاقة يؤثر على مادة (أنظر شكل B4).

مادة تحول حقل الطاقة الى حقل طاقة أخر (أنظر شكل c4).c4).

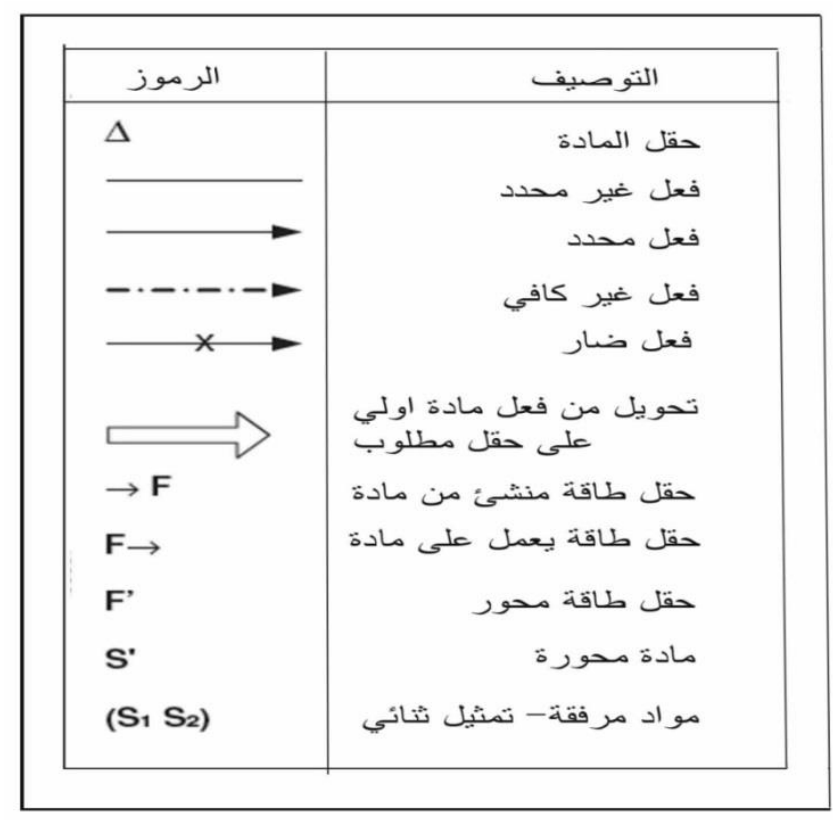

الشكل(3 ) ترجمة الباحث (Sivaloganathan؛ $(2000$

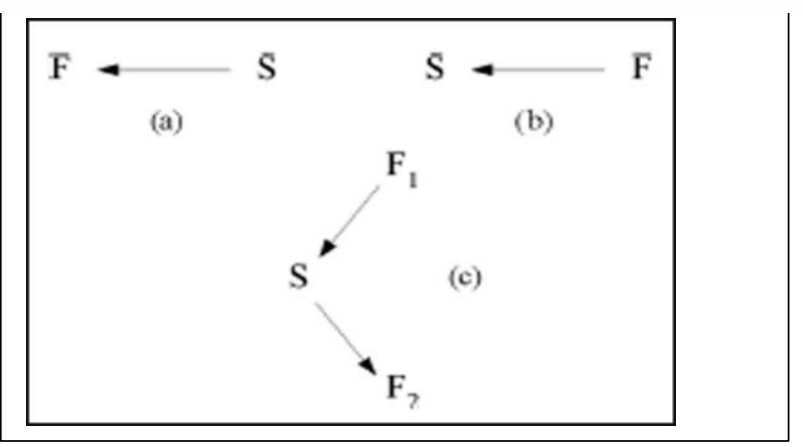

عند رسم مخطط حقل المادة يجب أن تكون المادة (S1,S2) في حقل المادة تتفاعل مع الطاقة هذا

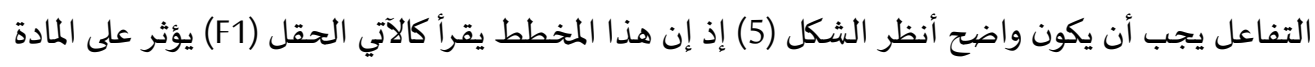

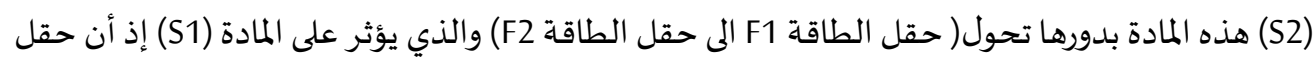


تطبيق أنموذج حقل المادة في حل مشاكل تصياميم المنتجات الصناعية.........محمد علي حسين القيسي ISSN(Online) 2523-2029, ISSN(Print) 1819-5229 2021 مجلة الأكاديي-العدد 101-السينة

الطاقة (F2) ممكن أن يكون نفس نوع الطاقة للحقل (F1) أو أن يكون هناك تحويل للطاقة من قبل (S2) لتكوين حقل الطاقة (F2) والذي هو عبارة عن شكل آخر من أشكال الطاقة إذ يُعد تحويل الطاقة من شكل فئل الى آخر خلال حقل المادة (Su-Field) هو الأكثر أهمية في حل المشكلات التقنية وكمثال على تحويل الطاقة

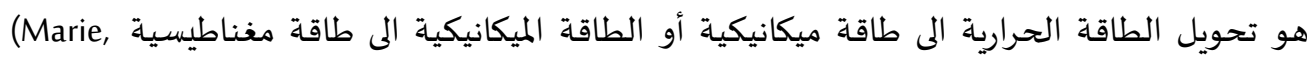
.2013)(

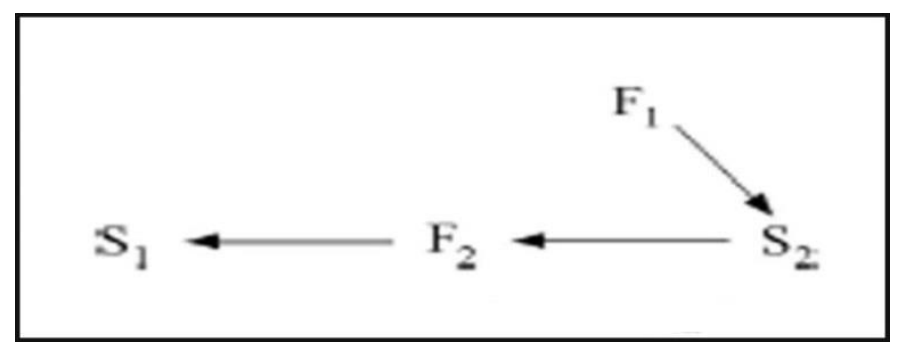

شكل (5) ترجمة الباحث (Marie، 2013)

وفيما يلي مثالين لتمثيل حقل المادة:

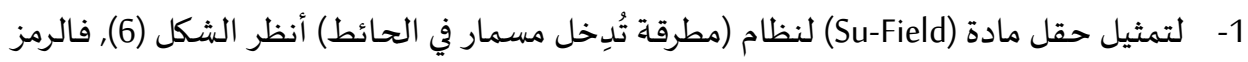
يمثل الطاقة الميكانيكية التي تعمل على المطرقة (S2) والتي بدورها تحول تلك الطاقة الى مادئ (F)

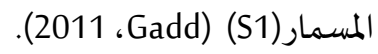

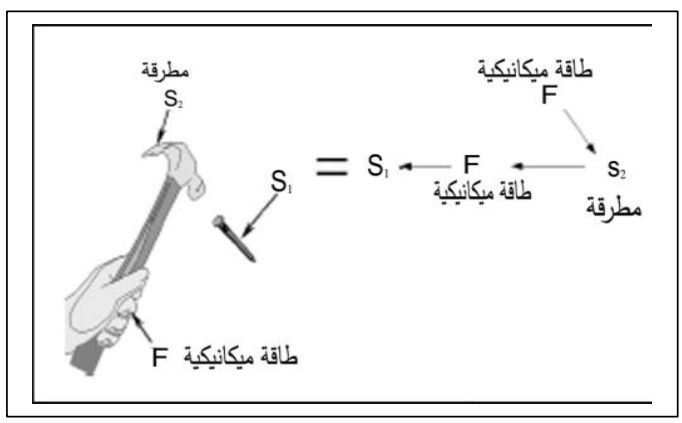

الشكل (6) ترجمة الباحث

2- مثال على تمثيل فعالية في نظام فمثلاً تكون الفعالية ( شخص يصبخ الحائط) فيكون التمثيل في

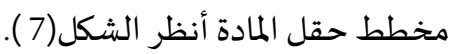

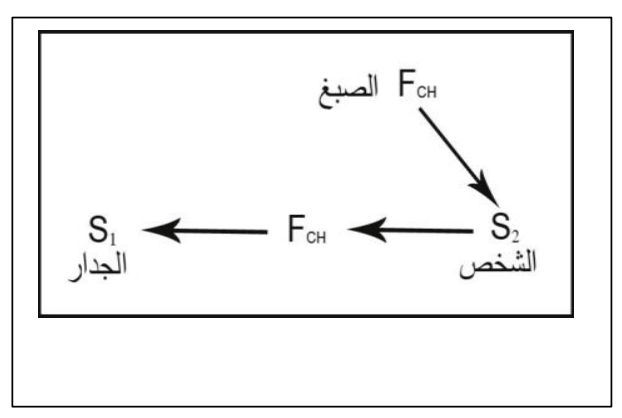

$$
\begin{gathered}
\text { = S1 } \\
\text { = S2 }
\end{gathered}
$$

FcH الصبخ (حقل كيمياوي) (Gadd, 2011, p. 34).

الشكل (7) ترجمة الباحث (Gadd، 2011) 
تطبيق أنموذج حقل المادة في حل مشاكل تصياميم المنتجات الصناعية.........محمد علي حسين القيسي

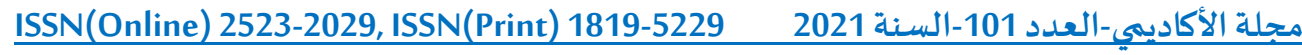

إجراءات البحث

منهج البحث: اعتمد الباحث على المنهج التحليلي التجريبي لغرض الوصول الى النتائج

مقدمة - مقدة

اختار الباحث مشكلة قائمة عند الكثير من المستخدمين في مجال الحدائق المنزلية إذ يعاني الأفراد من الصعوبة في نقل أو زراعة أو ازالة النباتات في الحديقة المنزلية باستخدام المجرفة اليدوية التقليدية

خصوصا في حالة التربة الصلبة التي تحتوي على جذور كثيفة من نفس النبات أو النباتات المجاورة.

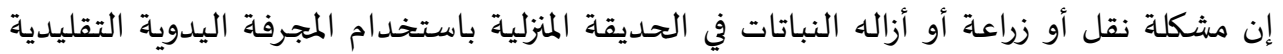

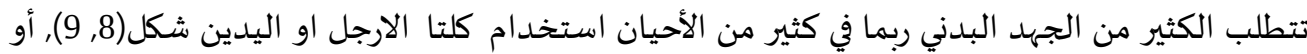

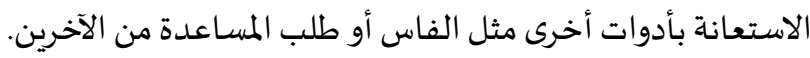

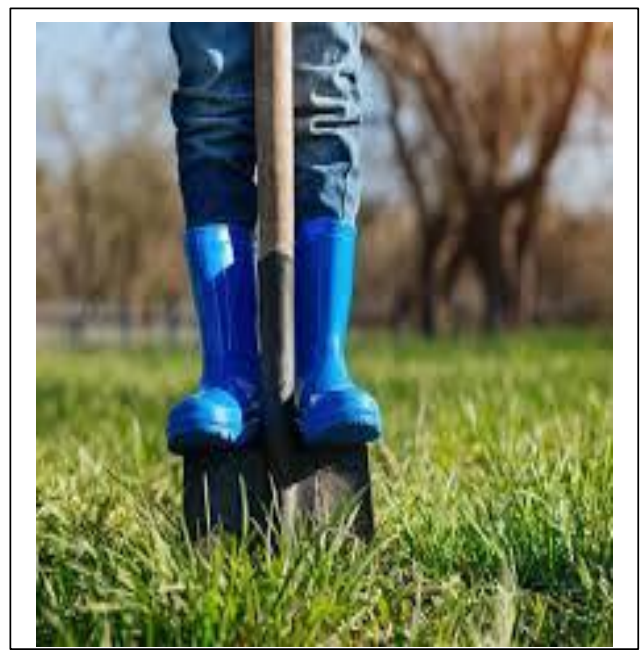

شكل(9)استخدام كلتا الارجل للمساعدة في غرس المجرفة بالارض, المصبدر جوجل

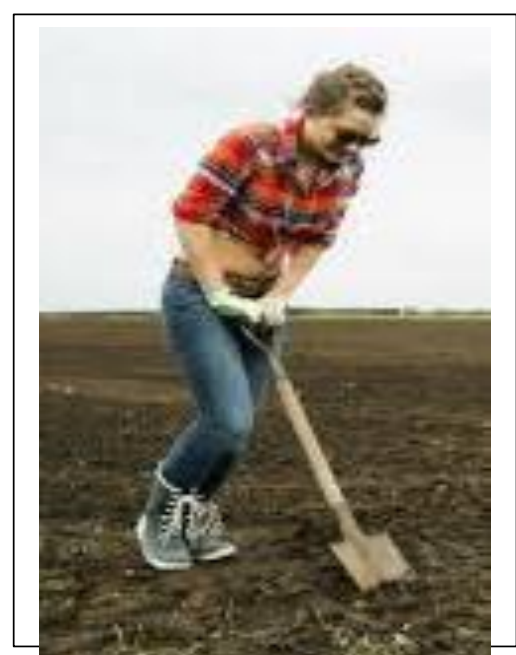

شكل(8)استخدام كلتا اليدين للمساعدة في غرس المجرفة بالارض المصددر جوجل

تحليل مشكلة الدراسة وحلها المجرفة

المجرفة بشكل عام هي أداة لحفر أو رفع أو نقل المواد المختلفة مثل قلع أو نقل النباتات او جذورها أو

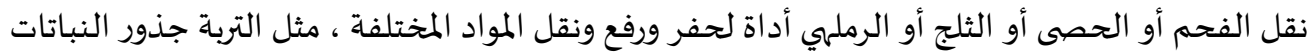

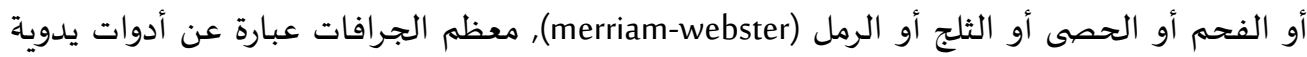

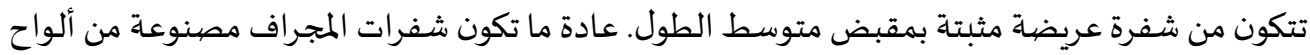

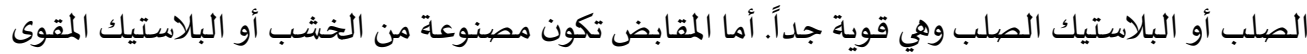

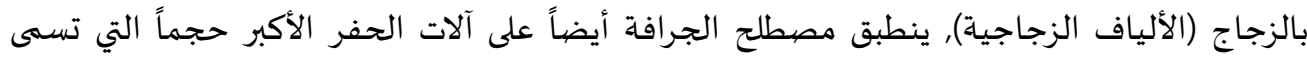
المجارف الآلية ، والتي تخدم نفس الغرض, يتم تكييف المجارف اليدوية للعديد من المهام والبيئات المختلفة. يمكن تخصيصها لمهمة واحدة أو تصيميمها على أنها متعددة المهام كما. إنها مفيدة جداً في الزراعاة. 
تطبيق أنموذج حقل المادة في حل مشاكل تصياميم المنتجات الصناعية.........محمد علي حسين القيسي ISSN(Online) 2523-2029, ISSN(Print) 1819-5229 2021 مجلة الأكاديي-العدد 101-السينة اما المجرفة قيد الدراسة فهي أداة لحفر الحديقة المنزلية لغرض نقل أو أزاله النباتات من مكان الى آخر

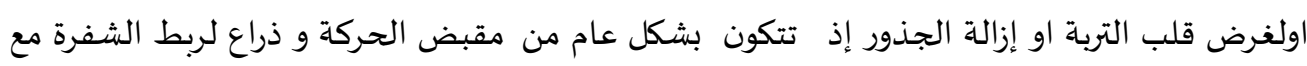

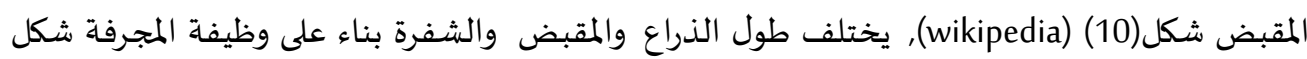

الأجزاء الرئيسية المجرفة

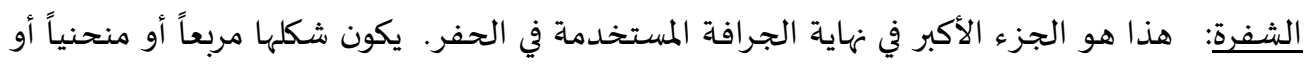

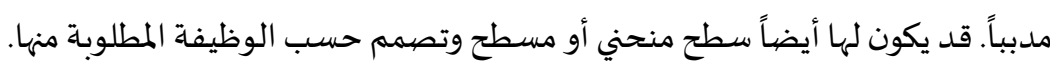

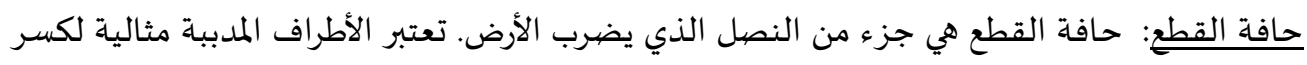

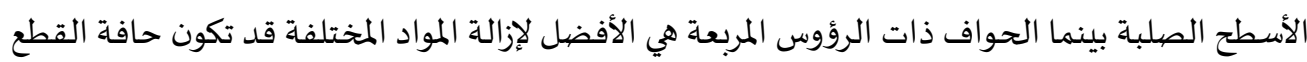

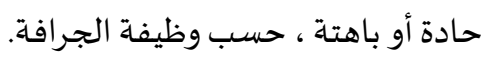

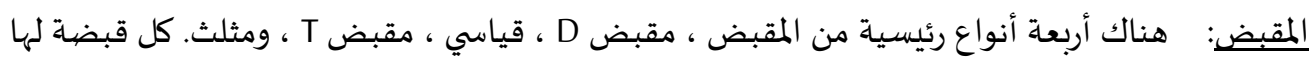
فوائدها الخاصية وتؤثر على كيفية عمل الجرافة ضيمن مجال عملها.

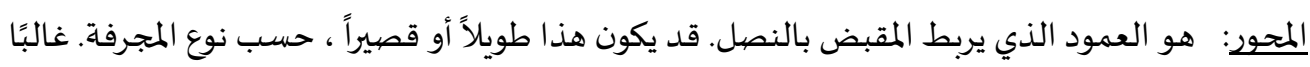

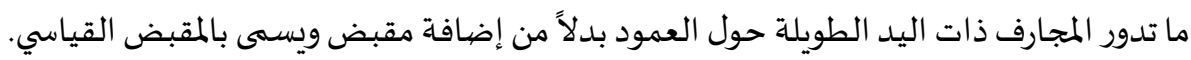

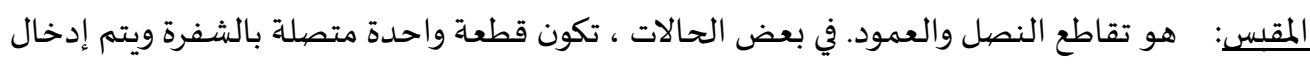

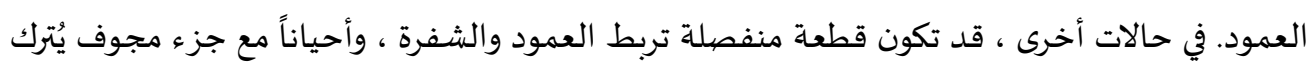
عمداً في نهاية العمود. ت فالات الخطوة: غالباً ما يكون الجزء الخلفي من الشفرة (ولكن ليس دائماً) سطحاً مسطحاً. تسمح هذه الخطوة

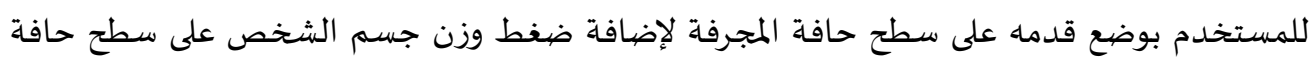

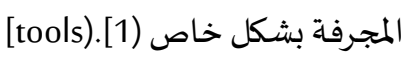

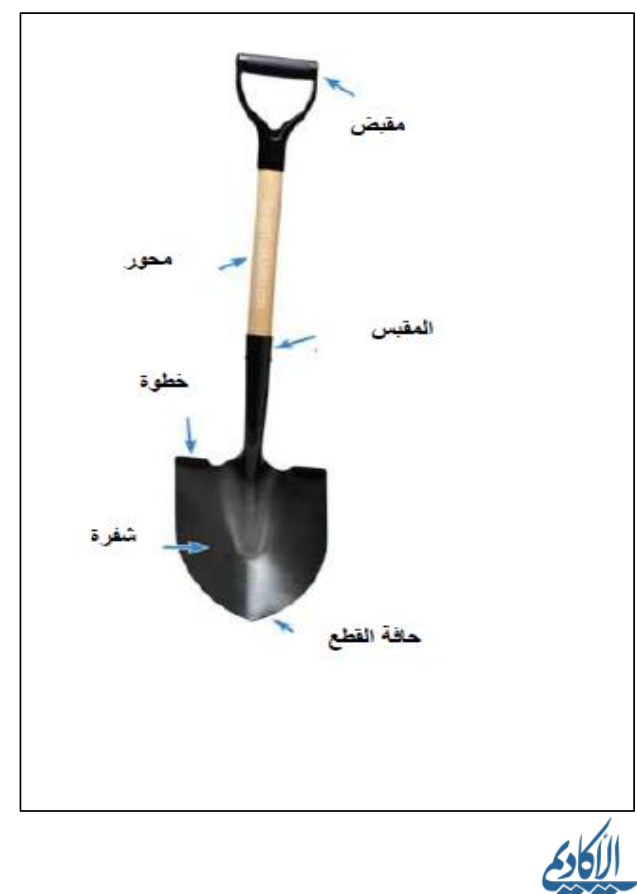

شكل(10) أجزاء المجرفة الأساسية (tools) 
تطبيق أنموذج حقل المادة في حل مشاكل تصياميم المنتجات الصناعية.........محمد علي حسين القيسي ISSN(Online) 2523-2029, ISSN(Print) 1819-5229 2021 مجلة الأكاديي-العدد 101-السينة

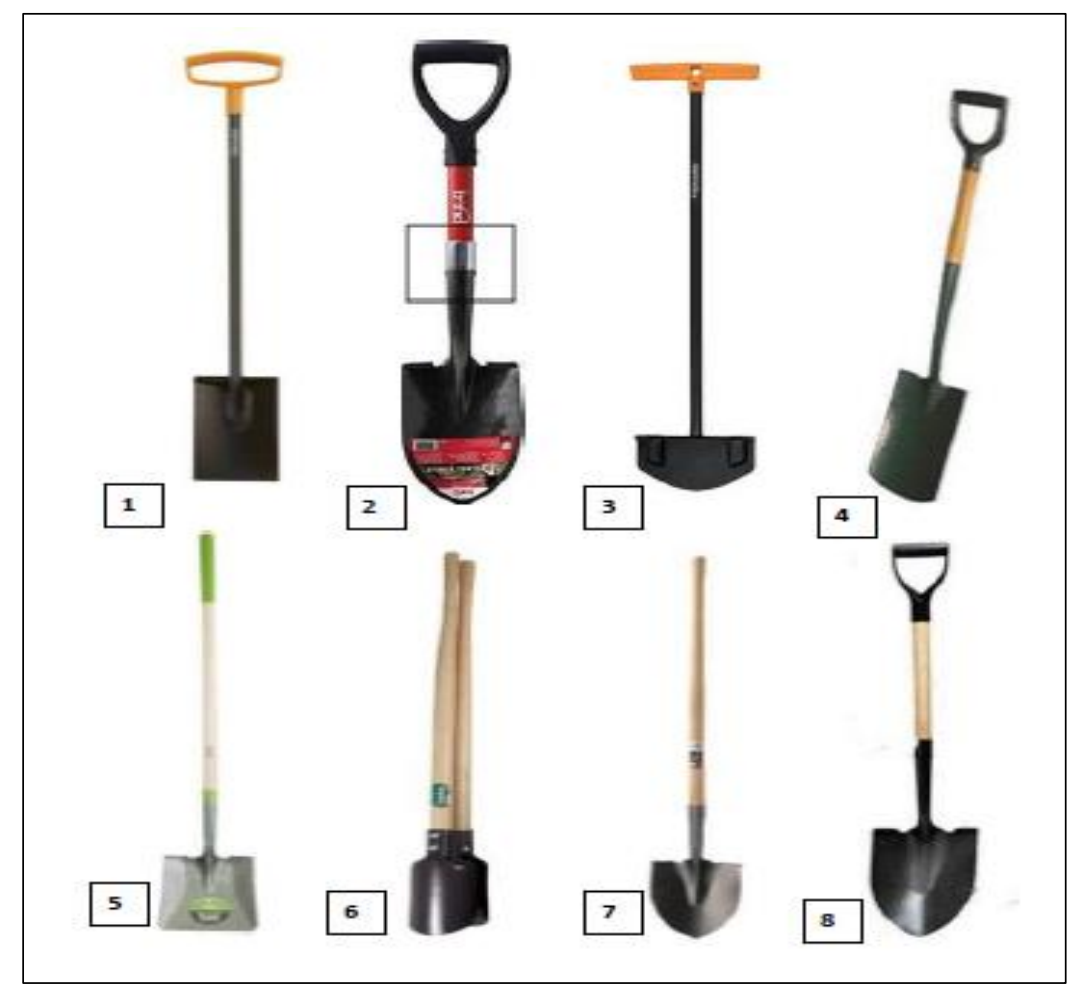

شكل(11) نماذج مختلفة من المجارف بناءً على وظيفتها (tools)

طريقةعمل المجرفة

1 - الطريقة التقليدية لعمل المجرفة المجرفه

إن الوصف التقليدي لعمل المجرفة مبين في شكل (A-B12 ,) إذ يتم تحريك المقبض يميناً ويساراً (افقياً) للحصول على توازن اتجاه الضغط من الرجل على شفرة المجرفة وبعد عدد من الضغطات يدات يدل

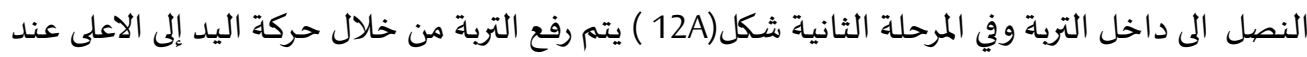
الذراع و حركة اليد الاخرى على المقبض الى الأسفل ليتم رفع التربة.. 
تطبيق أنموذج حقل المادة في حل مشاكل تصياميم المنتجات الصناعية.........محمد علي حسين القيسي

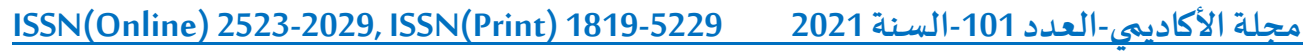

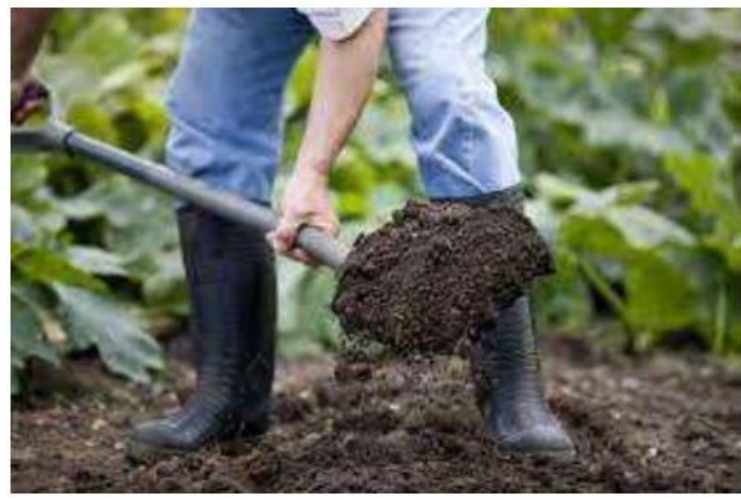

A

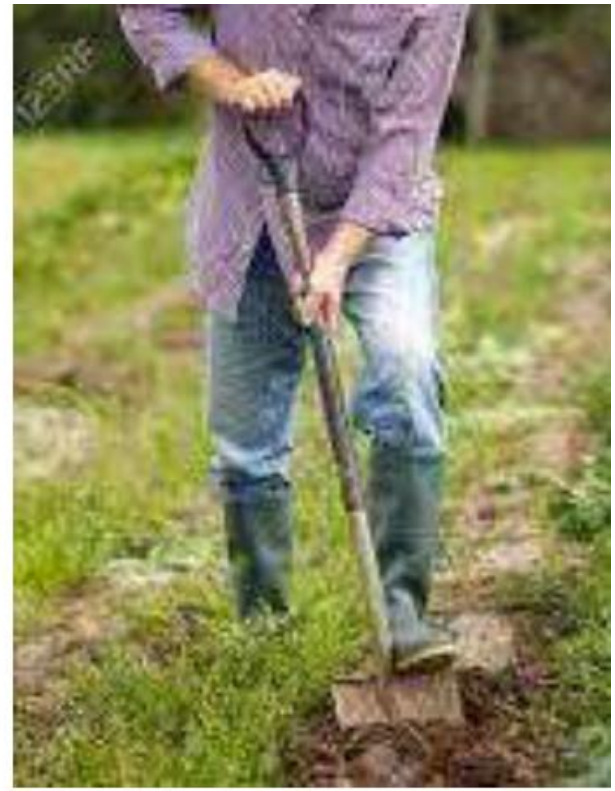

B

شكل (12) يبين الطريقة التقليدية لعمل المجرفة

لغرض تمثيل كيفية تحليل عمل المجرفة باستخدام حقل المادة انظر الشكل ( 13) والذي يتم على مرحلتين: اولاً غرس النصل في التربة: تتحرك اليد والقدم بالتناوب بغرض غرس المجرفة في التربة فالقوة

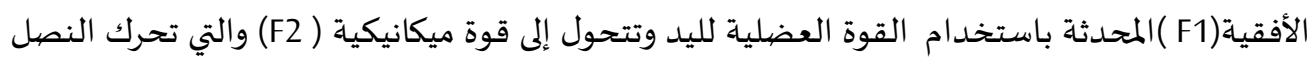

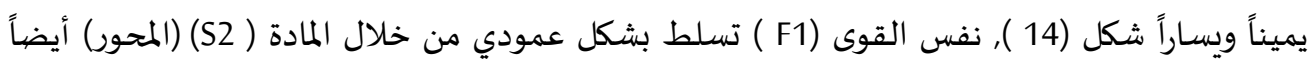

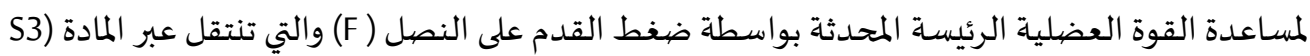

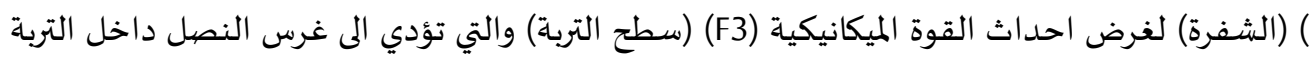

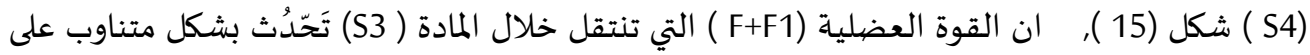

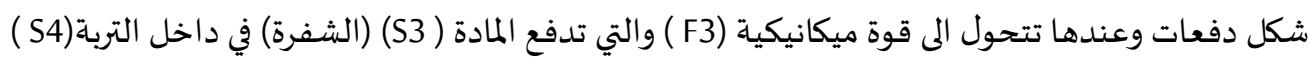


تطبيق أنموذج حقل المادة في حل مشاكل تصياميم المنتجات الصناعية.........محمد علي حسين القيسي ISSN(Online) 2523-2029, ISSN(Print) 1819-5229 2021 مجلة الأكاديي-العدد 101-السينة

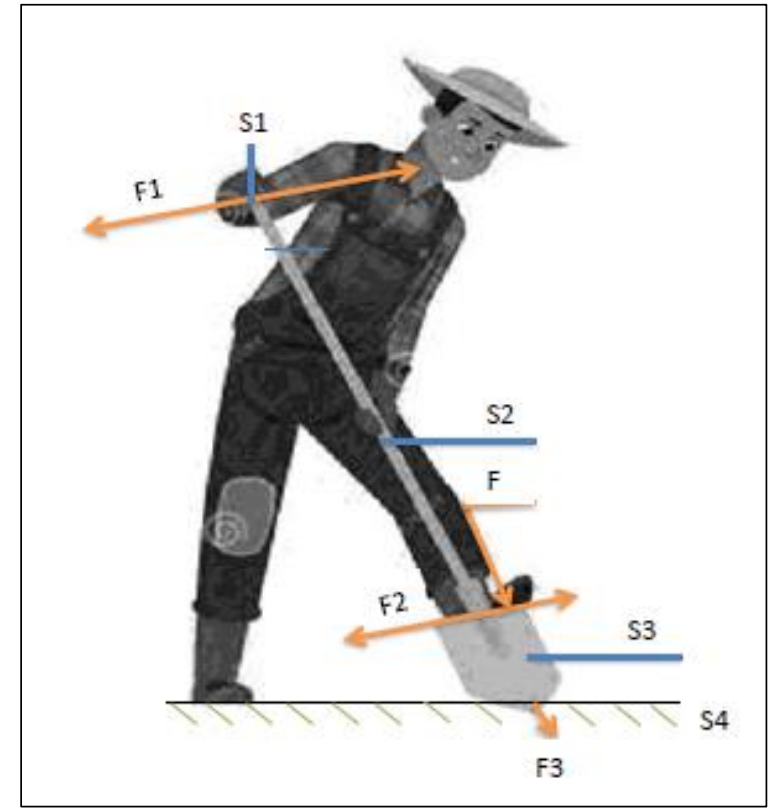

شكل(13) تمثيل عمل المجرفة (أثناء الغرس) حسب حقل المادة. إعداد الباحث
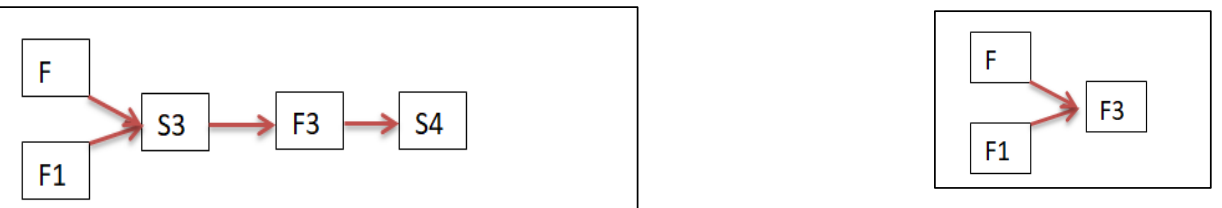

شكل(14) تمثيل العلاقات بين الطاقة والمادة, إعداد الباحث

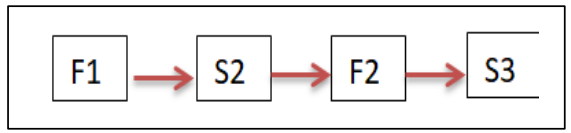

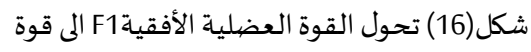

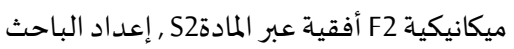

شكل(F1,F2) (F1) الى قوة ميكانيكية (F3) ).

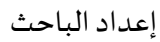

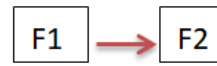

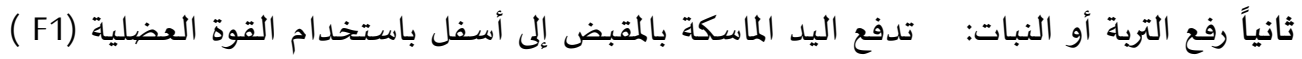

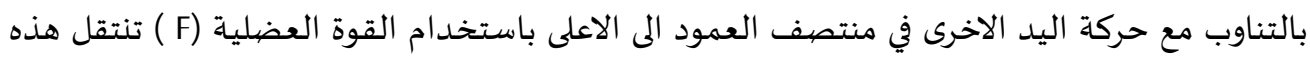

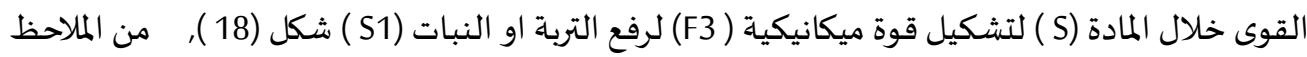

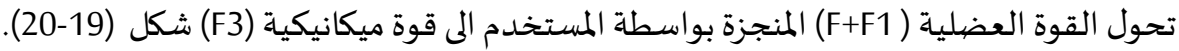


تطبيق أنموذج حقل المادة في حل مشاكل تصياميم المنتجات الصناعية.........محمد علي حسين القيسي

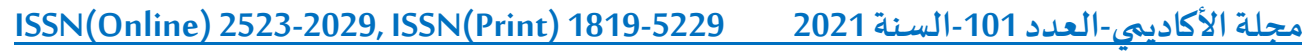

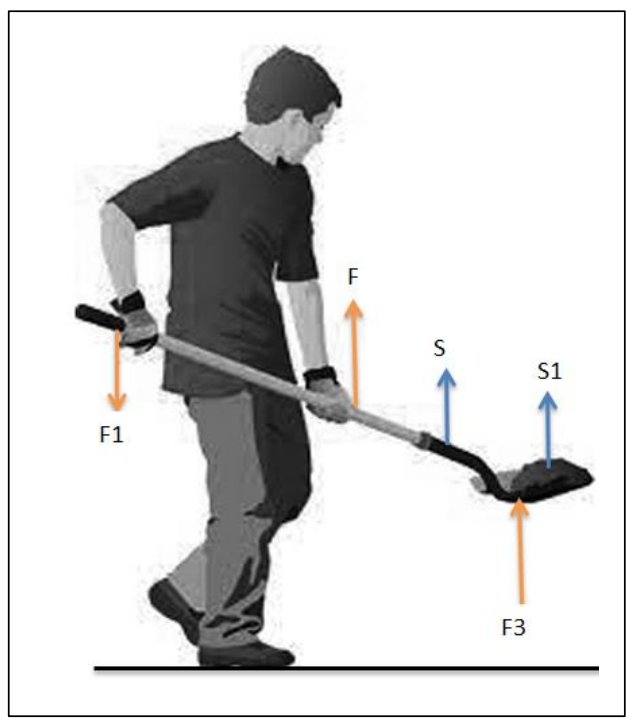

شكل(18) تمثيل عمل المجرفة (أثناء الرفح)

حسب حقل المادة المصيدر إعداد الباحث

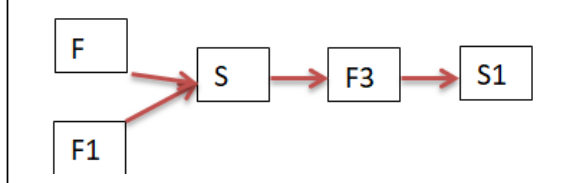

شكل(19) تمثيل العلاقات بين الطاقة والمادة(أثناء

الرفع) المصيدر تصميم وإعداد الباحث

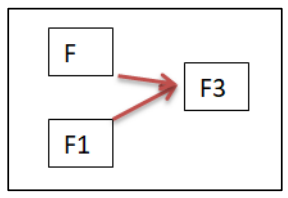

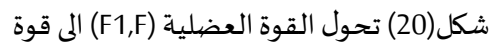

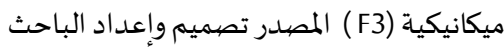

\section{مناقشـةتحليل النموذج}

من الملاحظ ان عمل المجرفة يكون على مرحلتين ,الاولى هي غرس النصل داخل التربة والثانية هي رفع

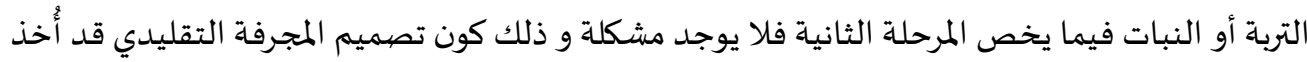

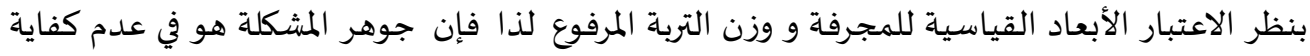

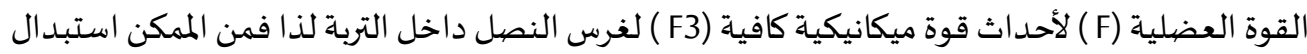

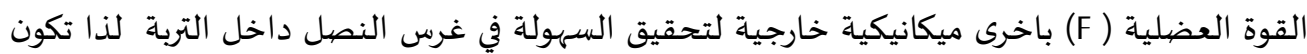

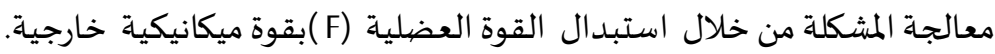
شكل(يمين 21) يبين مسار الطاقة ضمن المادة للتصيميم المقترح والذي يوفر القوة الميكانيكية المستبدلة من تحويل القوة العضلية الى قوة ميكانيكية كذلك فأن هذا الحل سوف يستغني عن القوة العضلية (F1) المسلطة من اليد على مقبض المجرفة شكل(يسار 21)
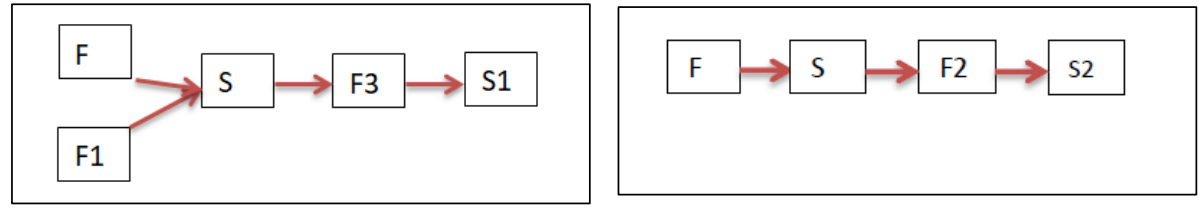

شكل(21)يمثل حقل المادة يمين للحل المقترح ويسار للحل التقليدي المصدر تصميم وإعداد الباحث 
تطبيق أنموذج حقل المادة في حل مشاكل تصياميم المنتجات الصناعية.........محمد علي حسين القيسي ISSN(Online) 2523-2029, ISSN(Print) 1819-5229 2021 مجلة الأكاديمي-العدد 101-السينة و لغرض الحصول على قوة ميكانيكية مشابهة للقوة العضلية على شكل دفعات فمن الممكن للقوة

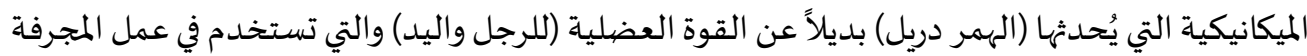
التقليدية لذا يكون مقترح التصميم كما في شكل (22يمين ABC), أما شكل (22) يسيسار (ABC ) فيبين العلاقة

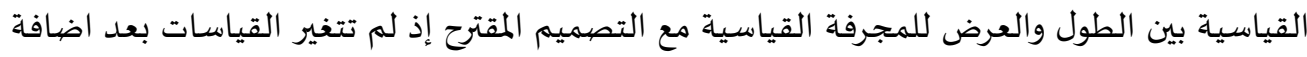

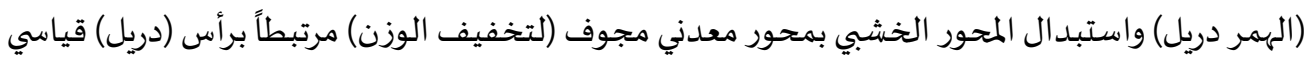

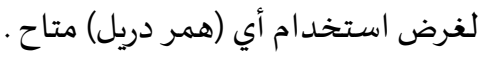

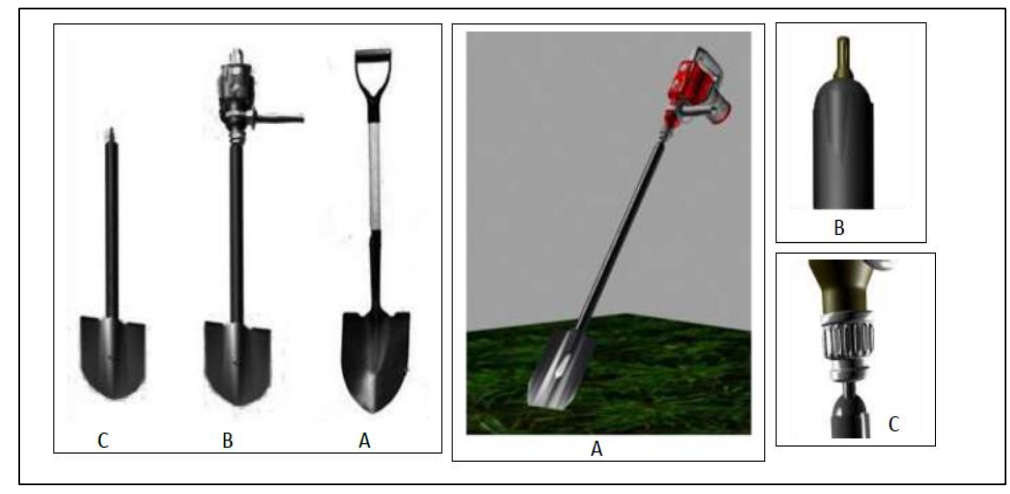

شكل(22) , المصدر تصميم وإعداد الباحث بأستخدام برنامج 3dsmax

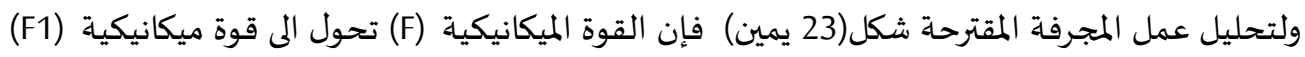

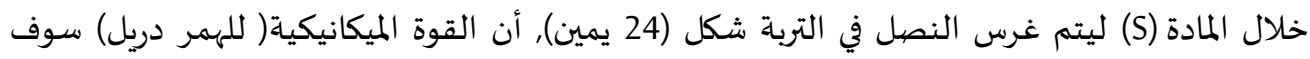

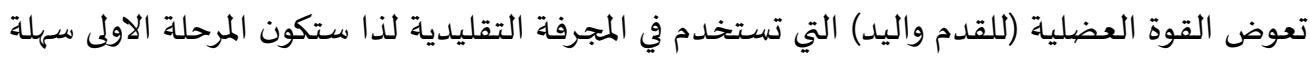
وسلسـة ولا تتطلب جهرد.

أما المرحلة الثانية وهي رفع التربة المى الاعلى لنقلها إلى مكان آخر موضحة في شكل (يسار 23), ان عملية رفع التربة في التصميم المقترح سوف تكون اسهل من رفع التربة في التصميم التقليدي إذ يتطلب الاخير

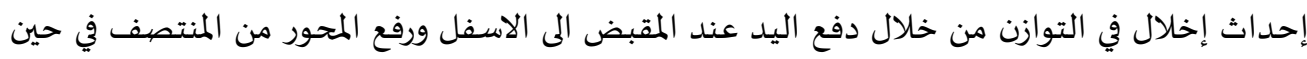

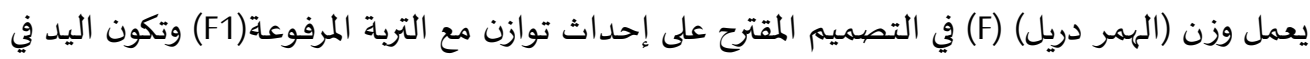
المنتصف هي مركز التوازن لذا لا يتطلب جهد في رفع التربة شكل(يسار 24).

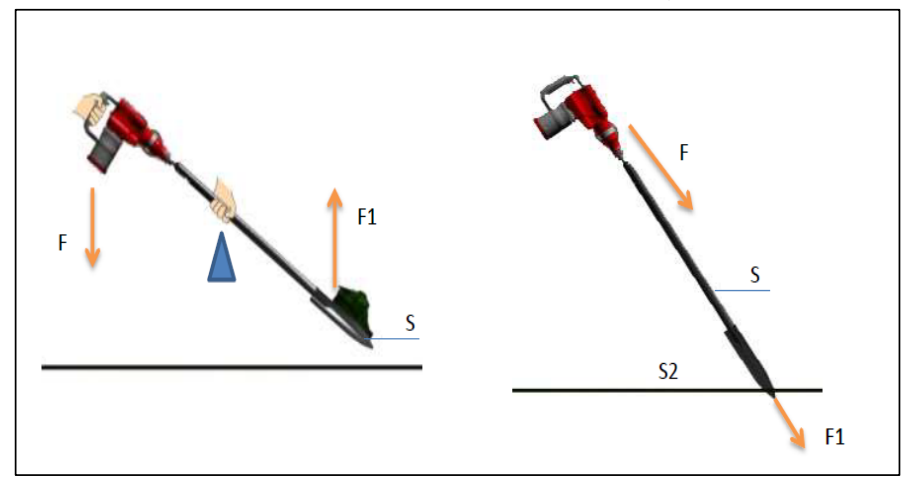

شكل(23) يمين غرس النصل داخل التربة يسار رفع التربة, المصيدر تصيميم وإعداد الباحث 3dsmax بأستخدام برنامج 
تطبيق أنموذج حقل المادة في حل مشاكل تصياميم المنتجات الصناعية.........محمد علي حسين القيسي ISSN(Online) 2523-2029, ISSN(Print) 1819-5229 2021 مجلة الأكاديي-العدد 101-السينة
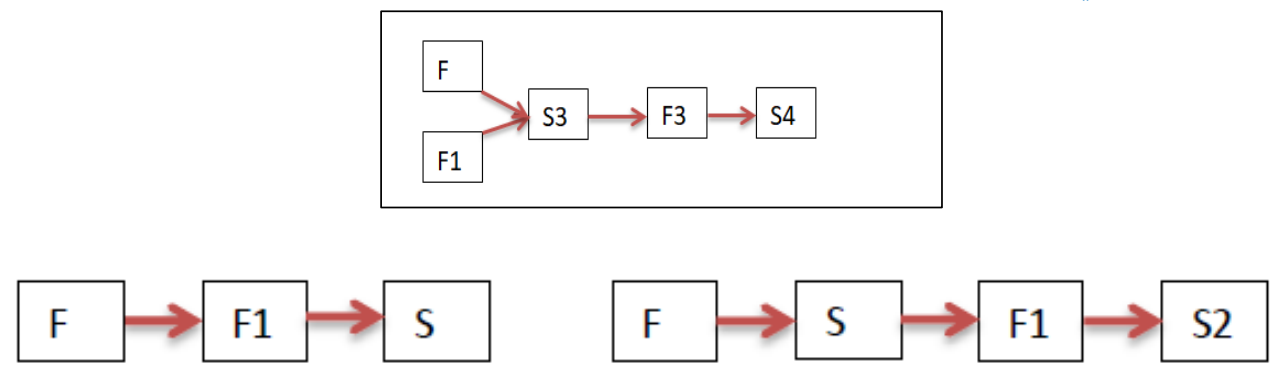

شكل (24) يمثل حقل المادةريمين غرس النصل داخل التربة يسار رفع التربة, المصدر تصميم وإعداد الباحث

\section{نتائج تحليل النموذج}

1- إن التصيميم المقترح يختصر الوقت والجهد المبذول في إنجاز أعمال الحديقة المنزلية.

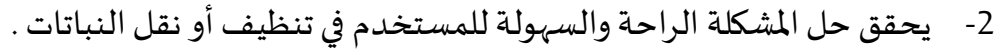

3- من الممكن ان يمتلك الشخص رأس المجرفة المحور فقط واستخدام (همر دريل) اذا توفر لدياء أو

استئجاره بمبلغ زهيد.

4- حل المشكلة سوف يريح المستخدم من استخدام القوة العضلية و أن الإفراط بها في أغلب الأحيان قد يتسبب له بأضرار في الجسم والعضلات.

5- لا يتطلب أجهزة او معدات إضافية أو الاستعانة بالآخرين بغرض انجاز العمل اذ من الممكن إنجازه

بشكل مفرد.

نتائج الدراسـة

1- تساهم إجراءات و نتائج بحثنا في تأسيس أرضية تحليلية سهلة يمكن تبنيها في حل مشكلات

$$
\text { تصميمية لاحقة. }
$$

2- إن حل مشكلة المجرفة يبين أهمية استخدام حقل المادة في حل مشكلات المنتجات الصناعية.

\section{الاستنتاحات}

1- من الممكن نقل الآليات الابتكارية في حل المشاكل من حقل هندسي صرف الى حقل التصيميم

$$
\text { الصناعي. }
$$

2- من الممكن تبسيط الآليات والمناهج الابتكارية المختلفة من حقول هندسية لتكون في متناول

$$
\text { المصيمم الصناعي لغرض استخدا مها. }
$$

التوصيات

1- يوصي الباحث بادخال آلية حقل المادة في المناهج الدراسية في التصميم التطبيقي الثلاثي الابعاد

$$
\text { مثل التصميم الصناعي والداخلي والعمارة. }
$$

2- يوصي الباحث المصيممين الصناعيين المحترفين على استخدام هذه الآلية في حل مشاكل التصميم الصناعي المختلفة لتكون شـائعة في متناول المصهمين الصنياعيين الجـدد وطلاب 
تطبيق أنموذج حقل المادة في حل مشاكل تصياميم المنتجات الصناعية.........محمد علي حسين القيسي

ISSN(Online) 2523-2029, ISSN(Print) 1819-5229 2021 مجلة الأكاديي-العدد 101-السينة

\section{References:}

Wikipedia. (2010).

Abbas, J. A.-K. (2012). The importance of tools in the technique of wood carving in contemporary Iraqi sculpture. Al-Academy Journal, 91.

Alqaisi, M. A. (2011). Employing the Arabic letter in industrial products using the 3dsmax program. Al-Academy Journal, 57.

alqaisi, M. a. (2020). Evaluate industrial products emotionally using emoji. Al-Academy Journal, 295.

Altshuller, G. (2006). Theory of inventive problem solving. DMC Otono.

Gadd, K. (2011). Triz For Engineers Enabling Inventive Problem Solving. UK,: John Willey \& Sons Ltd,.

Hussein, A. K. (2010). Function of color in industrial products. Al-Academy Journal, 31.

Hussein, A. K. (2015). The importance of social acceptance of inclusive design. Al-Academy Journal, 185.

Marie, G. (2013). Technology Forecasting(TF) using Hybrid Tech Mining, TRIZ TF For Research. USA,: North Carolina State University.

merriam-webster. (n.d.). Retrieved from shovel: https://www.merriam-

webster.com/dictionary/shovel

Michael. (2010). Innovation using the theory of inventive problem solving and select psychological methods. San Diago: California.

Nirjar, A. (2011). Entrepreneurship development. India,: Word Press,.

others, M. K. (2006). Mechanical Engineers HandbookMechanica /Volume 1, third edition. John Wiley \& Sons.

Rivin, V. F. (2005). Innovation on Demand. Cambridge University Press, 478.

Rivin, V. F. (2005). Innovation on Demand. Cambridge University Press.

Salamatov, Y. (2005). TRIZ:The right solution at the right timeA guide to innovative problem solving 2-nd edition. Institute of linnovative design.

Sivaloganathan. (2000). Design for Excellence. UK,: Cromwell Press,.

wikipedia. (n.d.). Retrieved from Shovel: https://en.wikipedia.org/wiki/Shovel 
DOI: https://doi.org/10.35560/jcofarts101/227-242

\title{
Applying the substance-field model mechanism to problem solving in industrial product design Mohammed Ali Hussein Alqaisi ${ }^{1}$
}

\begin{abstract}
:
Problem solving methods and mechanisms contribute to facilitating human life by providing tools to solve simple and complex daily problems. These mechanisms have been essential tools for professional designers and design students in solving design problems.

This research dealt with one of those mechanisms, which is the (the substance-field model model), as it has been mentioning that this mechanism is characterized by the difficulty of its application, which formed the main research problem. In home gardens (the sub-problem of research), an analysis of this problem was applied and then a solution was found to address it. The researcher used the $3 \mathrm{~d}$ smax program to implement the proposed design.

The most important research results were: .

1- The research methodology and the method of applying the substance-field model mechanism to solve a problem can be applied to solve various simple and complex design problems by professional designers in addition to its importance for design students in particular.

2- The research presented a proposed model to solve the problem of the shovel using the substance-field model mechanism.
\end{abstract}

Keywords: substance-field, shovel, Theory (TRIZ)

\footnotetext{
${ }^{1}$ College of Fine Arts/ University of Baghdad, mohammed.ali@cofarts.uobaghdad.edu.iq .
} 